\title{
ROCKING OF MULTIBLOCK STONE CLASSICAL COLUMNS
}

\author{
DORA FOTI \& VITANTONIO VACCA \\ Department of Civil Engineering Sciences and Architecture, Polytechnic University of Bari, Italy
}

\begin{abstract}
This paper investigates the dynamic behaviour of the simplest structural element, the classical column, considered as a monolithic multiblock. In particular, we studied the behaviour of these structures under harmonic pulses of variable amplitude and frequency, tracing the rocking curves that represent the "dynamic identity card" of such structures. These curves were plotted by varying the quantities that influence the dynamics of the system: slenderness of the column (total height ratio of the column, $\mathrm{H}$, and width of the base, B), the number of drums constituting the column, the contact properties (coefficient of friction, normal and tangential stiffness of the joint) and damping. The slenderness ratio range considered varies between $\mathrm{H} / \mathrm{B}=4$ to $\mathrm{H} / \mathrm{B}=13$, so as to cover all the columns belonging to the Doric and Ionic orders present in the Mediterranean basin. For the numerical analyses, it was used the Distinct Element Method (DEM) that, in the physics of multibody systems, allows us to study the dynamic response with high reliability of systems in which the size of the individual blocks can be compared with the overall size of the structure.
\end{abstract}

Keywords: multi-drum column, rocking, D.E.M.

\section{INTRODUCTION}

Medium-high intensity earthquakes can damage historical and monumental heritage, such as classical columns and columns of the most significant archaeological sites that are abundantly present in highly seismic areas of the Mediterranean basin such as Turkey, Syria, Greece, Cyprus and Southern Italy (Fig. 1). Multi-block columns are made up of blocks arranged one over each other, usually without unbound connection materials between them. The seismic behavior of these structures exhibits rocking, sliding, and twisting phenomena between the various blocks that clearly distinguish them from modern structures.

In the present paper in order to describe the behavior of the multiblock columns, the so-called distinct elements modeling has been used, particularly suitable for describing with high reliability the behavior of structures consisting of elements (blocks) of significant size with respect to the overall dimensions of the structure [1], but also of ancient monuments [2], or masonry elements [3].

The finite element method (FEM) can use interface elements to model the discontinuities in the material. However, the application of the interface elements is limited for several reasons (e.g. difficulties in implementation of many intersected interfaces and difficulties in the recognition of the evolution of the contacts; evaluation difficulties when recording large displacements, etc.) [4]-[9].

To overcome some of these restrictions, Distinct Element Method (DEM) was originally proposed in the early 70 s as a way to solve the problems of rock mechanics [10]. In this case, in fact, distinct elements were used to simulate dynamics problems of discontinuous rock masses. The method assumes a set of discrete blocks, where interfaces between the bodies (the joints) are called discontinuities and are treated as boundary conditions for the individual block. This method is usually used when the global behavior is dominated by discontinuities, i.e. the structure consists of a set of discrete blocks. 
The main features of DEM are:

- The system is constituted by a set of blocks that interact along the contact planes/points: the blocks do not have the possibility to interpenetrate and overlap;

- Discontinuities are considered as areas of interaction between the blocks: it is prescribed a model of behavior for these interactions;

- The method uses an explicit time algorithm that allows large rotations, displacements and linear or non-linear constitutive relations for both the block matrix and the discontinuities.

3D numerical analyses have been conducted using 3DEC software [11]. In the software, the blocks have been modelled like perfectly rigid blocks, while interactions between the different blocks are modelled with an interface with zero thickness, with a normal (JKn) and shear (Jfric) elastic stiffness. No cohesion has been considered. In the following the parameters adopted for modeling in 3DEC are defined:

- $\quad$ Density $=2200 \mathrm{Kg} / \mathrm{m}^{3}$

- Coulomb Contact law: Friction angle $\varphi=30^{\circ} \mid$ cohesion $\mathrm{c}=0$

- Contact properties: Normal stiffness $\mathrm{Kn}=10^{11} \mathrm{~Pa} / \mathrm{m} \mid$ Shear stiffness $\mathrm{Ks}=10^{11} \mathrm{~Pa} / \mathrm{m}$

- Damping: "local" damping $=0.5$

Regarding the loads applied, initially the column was loaded statically by its own weight. Thereafter, a sinusoidal or cosinusoidal harmonic law pulse was applied at the base.

The slenderness range considered varies from a minimum value, $\mathrm{H} / \mathrm{B}=4$, to a maximum one, $\mathrm{H} / \mathrm{B}=13$ [12], so as to cover all the columns belonging to the Doric and Ionic orders present in the Mediterranean basin.

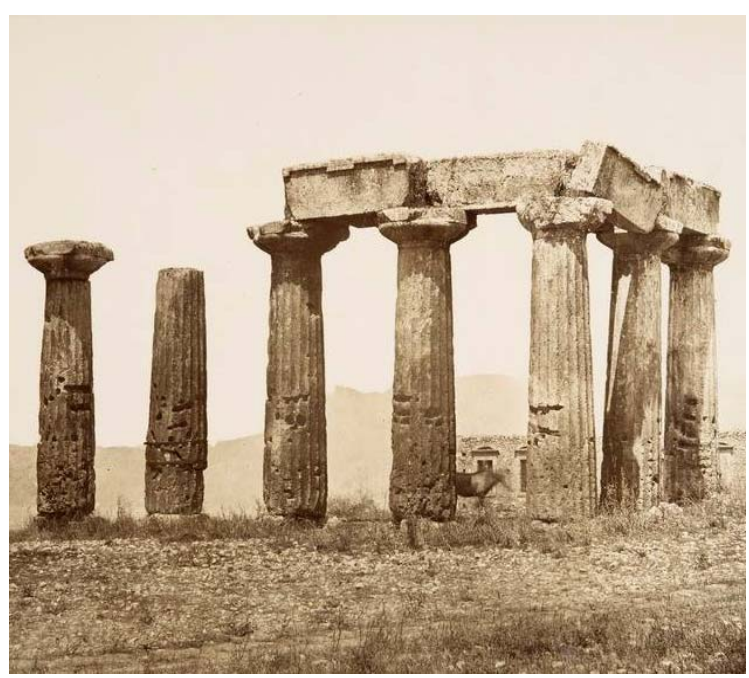

(a)

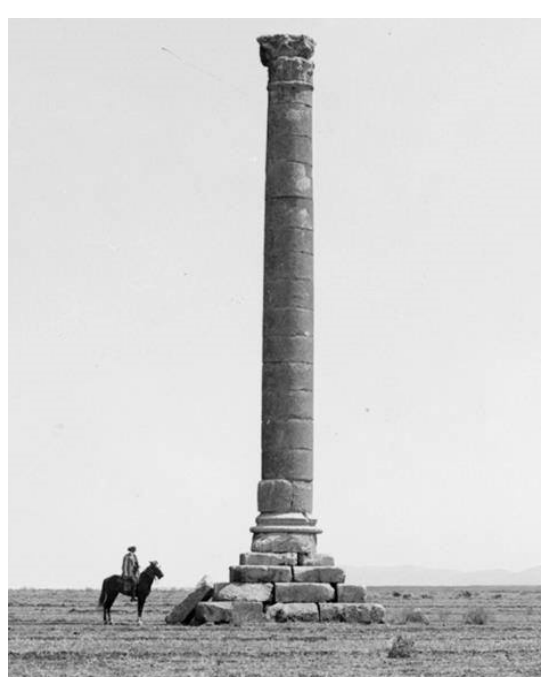

(b)

Figure 1: a) Corinth, Apollo's temple; (b) Taat, Libanon, Corinth column (Source: photo of early '900 - Royal Collection Trust/ her Majesty Queen Elizabeth II 2014). 


\section{RESULTS}

By striking the column with harmonic pulses, consistent with what was observed in [13], the following behaviour has been observed:

- The column keeps still and undamaged although it has recorded more or less evident displacements and rotations (Safe Area);

- The column collapses entirely after oscillating at least once around the base, i.e. the collapse occurs after the base of the column exhibits one or more impacts with the support surface (mode I);

- The column collapses entirely without oscillating around the base, i.e. without the base impacting the ground before the total collapse occurs (mode II);

- The column undergoes a partial collapse (mode III).

Showing the observed results in a frequency - peak acceleration plot of the harmonic pulse (Fig. 2) the stability/instability regions of a column can be defined as follows.

The red points identify the line of overturning without impact (mode II) and all points above this line represent the total collapse zone; the yellow points identify a region of overturning with impact (mode I), while the pink ones identify the partial collapse region that is clearly present only in the multiblock columns; all other points belong to the stability region for the column.

The safety zone in the frequency-peak acceleration plane is clearly dependent on the various parameters that govern the dynamics of the phenomenon, in particular: a) sizes, b) column slenderness ratio $\mathrm{H} / \mathrm{B}$, c) number of blocks composing the column, d) damping coefficient, e) contact properties between the blocks. Then, the results of impulsive analyzes are presented and discussed as the parameters governing the phenomenon of overlapping of multiblock columns vary.

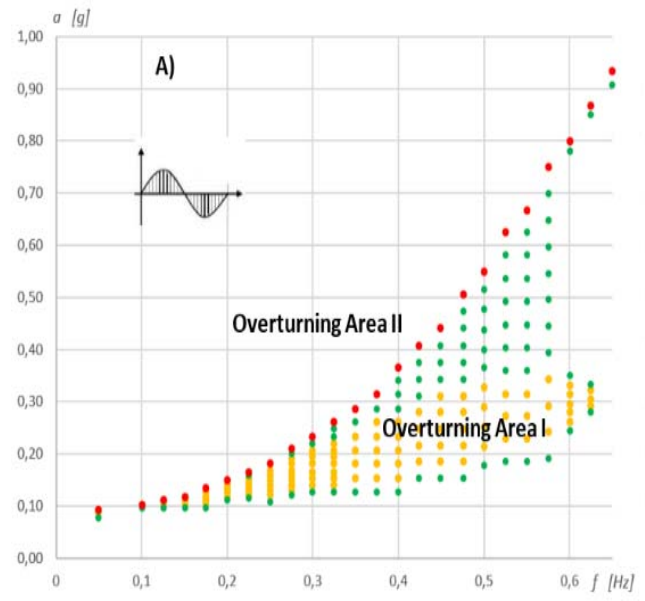

(a)

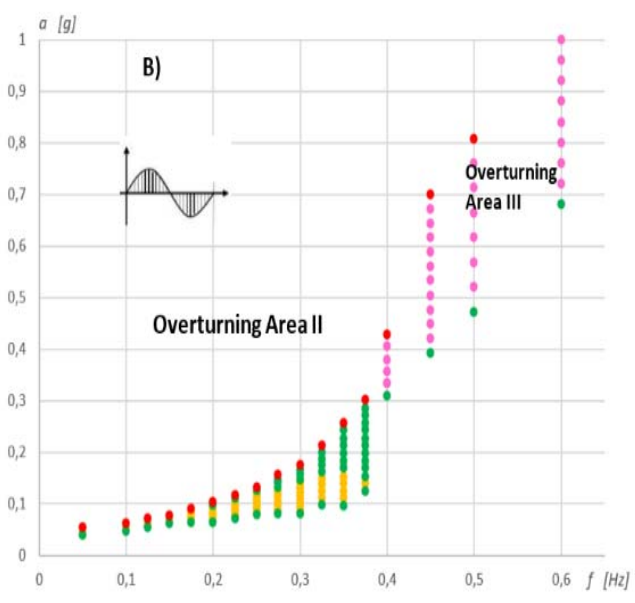

(b)

Figure 2: Frequency $[\mathrm{Hz}]$ - peak acceleration plot of the harmonic pulse $[\mathrm{g}]$ of a column with $\mathrm{H} / \mathrm{B}=4$ ratio subjected by a sinusoidal impulse at the base: (a) monlitic column; (b) column composed by 4 blocks. 
2.1 Safe area obtained by varying the slenderness $(\mathrm{h} / \mathrm{b})$ of the column

The slenderness of the column is defined as the ratio between the height, $\mathrm{H}$, and the diameter of the section of the imoscope, B, (bottom section of the base of the column). As previously mentioned, the columns belonging to the Doric and Ionic classical orders have been analysed with a slenderness ranging from 4 to 13 [12], consisting of a fixed number of blocks, $n_{R}$ (in this case equal to 8 ), with a base diameter $B=1 \mathrm{~m}$ and increasing heights, $\mathrm{H}(4-13 \mathrm{~m})$. In Fig. 3, the results of the analyses are reported.

In Figs 3(a) and 3(c) the plots in the frequency-peak acceleration plots are reported, respectively in $\mathrm{Hz}$ and in g; in Figs 3(b) and 3(d) the plots are reported on the same plane but with adimensionalised quantities and having indicated with $\mathrm{H}$ and $\mathrm{B}$, respectively, the height and diameter of the column's base, $g$ the acceleration of gravity, ag and $f$ the width in acceleration and the frequency of the applied harmonic pulse. In Fig. 4 the same curves reported in Fig. 3 are plotted in one graph.

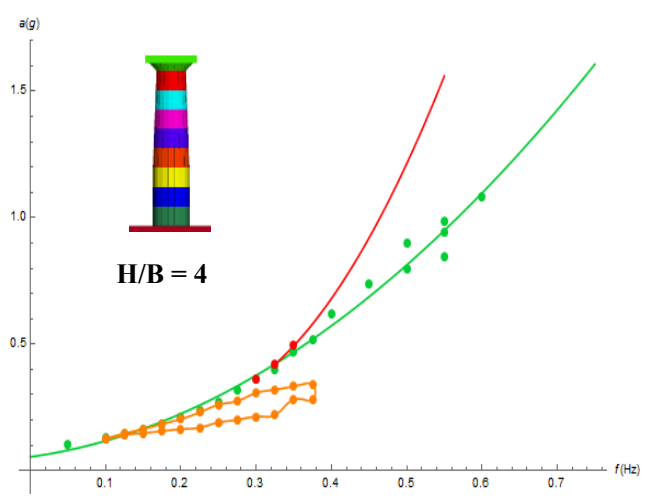

(a)

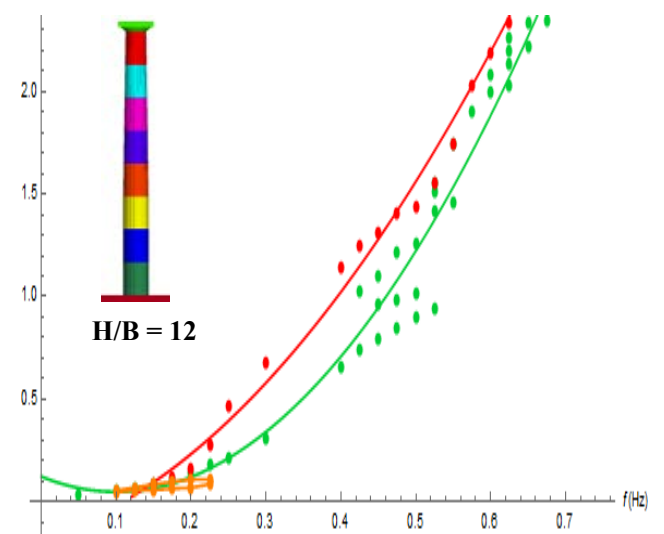

(c)

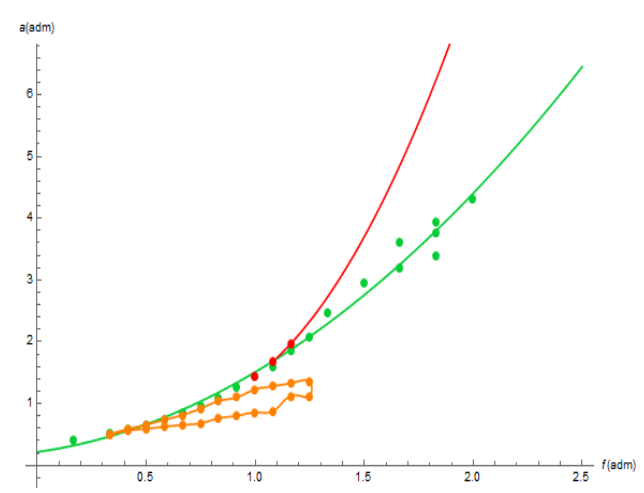

(b)

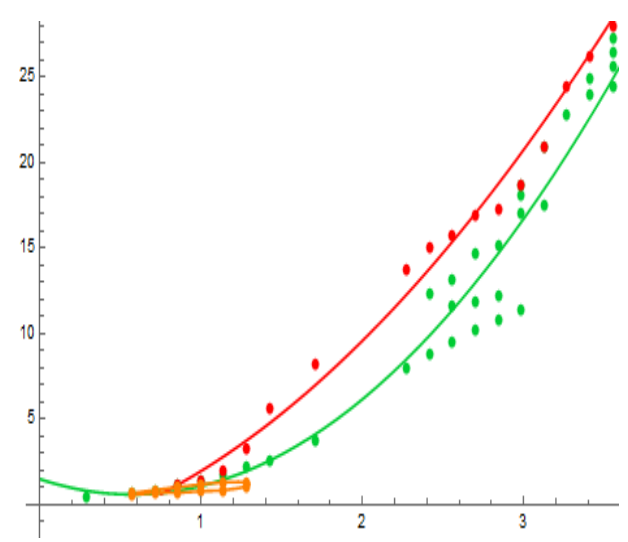

(d)

Figure 3: (a), (c) Frequency-peak acceleration plots of cosine-like harmonic acceleration pulse of a column with ratio $\mathrm{H} / \mathrm{B}$ equal to 4 and 12 subjected by a cosinusoidal acceleration impulse at the base: the column on the left shows the plots with dimensions on the axes, respectively, in Hzand in g; (b), (d) adimensionalised plots. 
The diagrams obtained in the dimensional plan show that the column stability region decreases as the H/B ratio increases, especially for low frequencies: for the column with slenderness $\mathrm{H} / \mathrm{B}=4$ at the frequency $\mathrm{f}=0.05 \mathrm{~Hz}$ there is an acceleration of collapse of 0.102 $\mathrm{g}$, while for the column with slenderness $\mathrm{H} / \mathrm{B}=13$ the collapse acceleration is reached for $0.037 \mathrm{~g}$ (almost three times lower). For every increasing frequency, the green curves tend to come close to each other, but globally always confirm the same order; the highest one is relative to the most squat column and, as far as the slimmer column is concerned, the curve is lowered.

The yellow curves reduce the area around them considerably as the slenderness increases, therefore, contrary to what can be expected, a squat column will be more sensitive to global collapse, after oscillation, than a slender column. Red curves appear at less frequencies and accelerations with increasing slenderness: for slenderness $\mathrm{H} / \mathrm{B}=4$ the bifurcation occurs for $\mathrm{f}=0.298 \mathrm{~Hz}$ while for $\mathrm{H} / \mathrm{B}=13$ a frequency $\mathrm{f}=0.150 \mathrm{~Hz}$ is recorded. Therefore, a region where partial collapses occur are registered, they increase with the increase of the slenderness of the column.

Adimensionalising the axes, it is immediately observed (Fig. 4) that all curves tend to overlap, thus showing invariant trends with respect to the slope $\mathrm{H} / \mathrm{B}$ of the column.

Similar analyses have been made by stressing the columns with sinusoidal pulses. Fig. 5 shows the results of the analyses.

Unlike the case with cosine-like pulse, the yellow curves describe significantly lower areas, so as not significant in the case of a column with $\mathrm{H} / \mathrm{B}=4$. In addition, the red curves disengage from the green ones at a frequency ranging from 0.10 to $0.15 \mathrm{~Hz}$, while in the former case they started with decreasing frequencies as the slenderness increased, in a wider frequency range $(0.15-0.35 \mathrm{~Hz})$. Even in this case, by adimensionalising the magnitudes, there is a superposition of curves at low frequencies, while at adimensionalised frequencies greater than 0.55 there are different trends.

\subsection{Safe area obtained by varying the global dimensions of the column}

It is well known that the global dimensions of structures, with the same geometric relationships, significantly affect their dynamic behaviour. The study of the variation of the

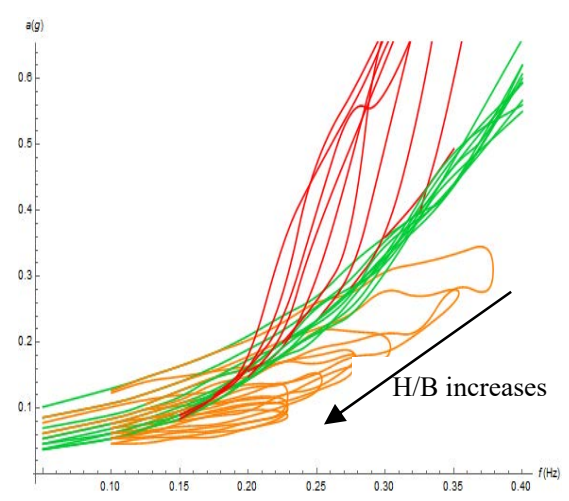

a)

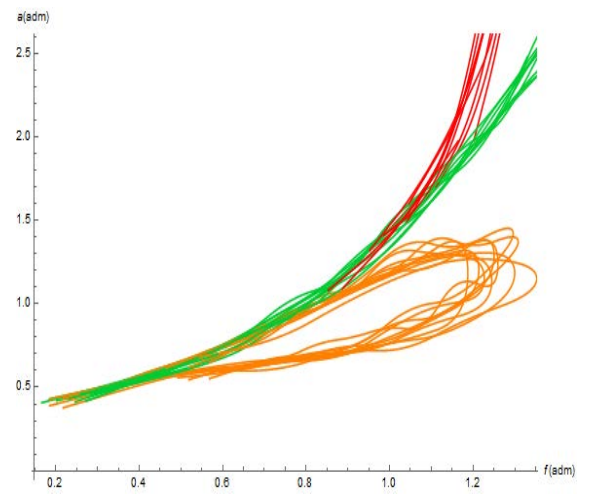

b)

Figure 4: a) Frequency-peak acceleration plot of cosine-like harmonic acceleration pulse of a column with a ratio $\mathrm{H} / \mathrm{B}$ variable from 4 to 13 , subjected by a cosinusoidal acceleration impulse at the base; b) adimensionalised plots. 


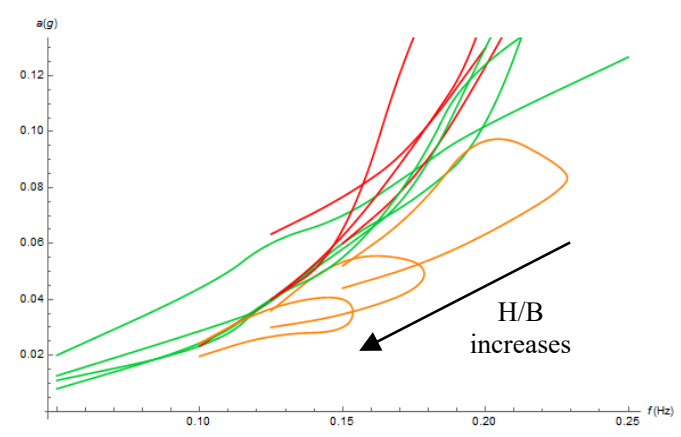

a)

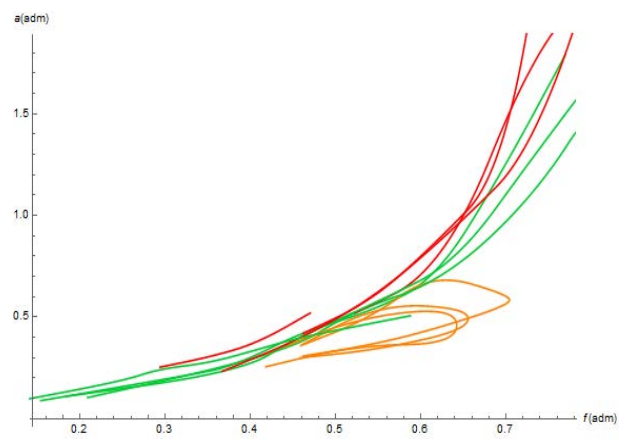

b)

Figure 5: a) Frequency-peak acceleration plot of sine-like harmonic acceleration pulse of a columns with ratio $\mathrm{H} / \mathrm{B}$ variable from 4 to 13 subjected by a cosinusoidal acceleration impulse at the base; b) adimensionalised plots.
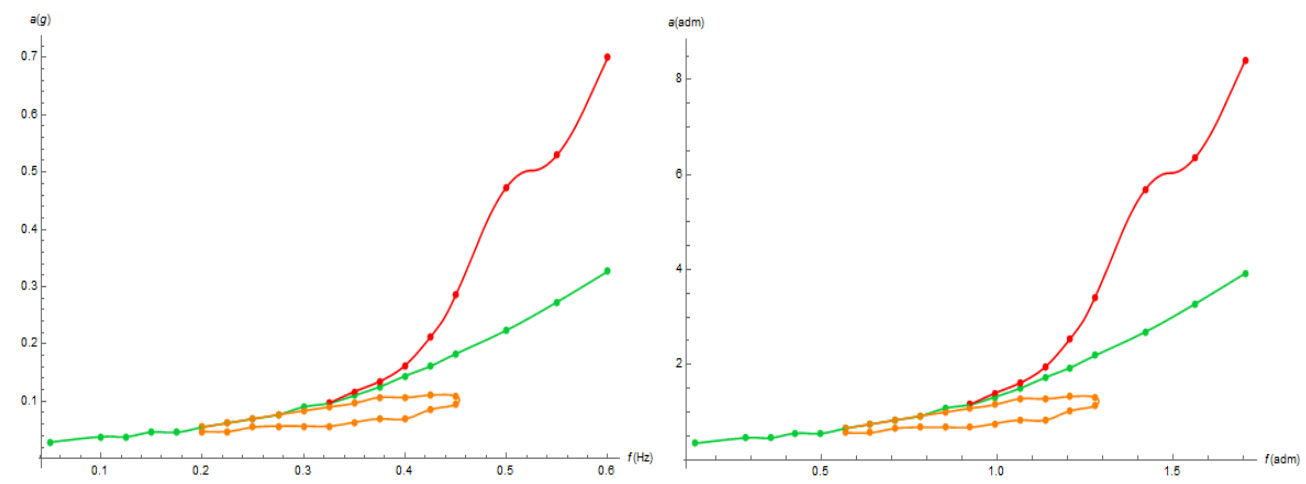

$\mathrm{B}=0.25 \mathrm{~m}$

a)

b)
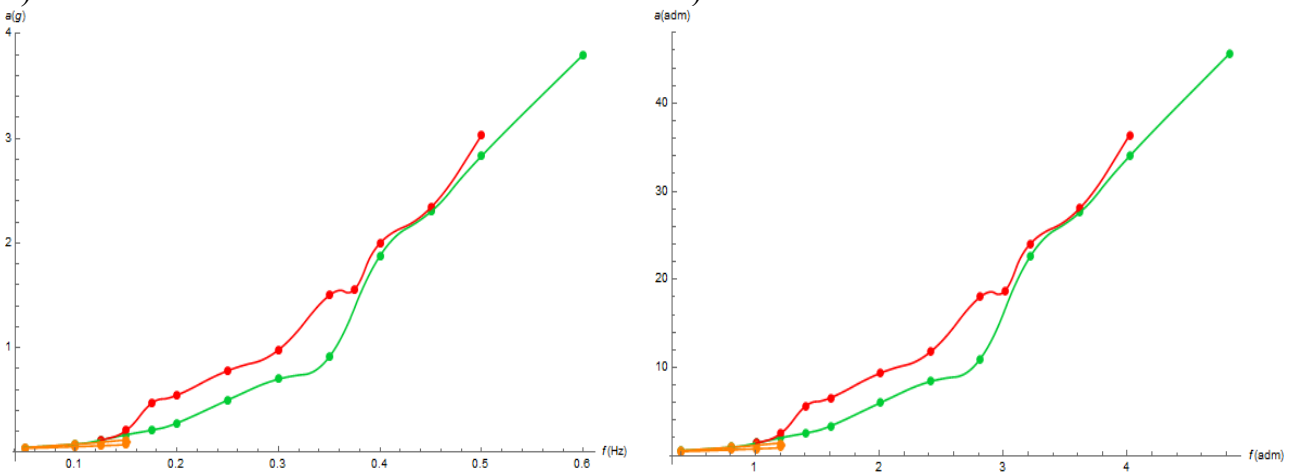

$\mathrm{B}=2.00 \mathrm{~m}$

c)

d)

Figure 6: a), c) Frequency-peak acceleration plots of cosine-like harmonic acceleration pulse of a column with $\mathrm{H} / \mathrm{B}=12,8$ blocks and base diameter variable from 0.25 to 2.00 $\mathrm{m} ; \mathrm{b}), \mathrm{d})$ adimensionalised plots. 


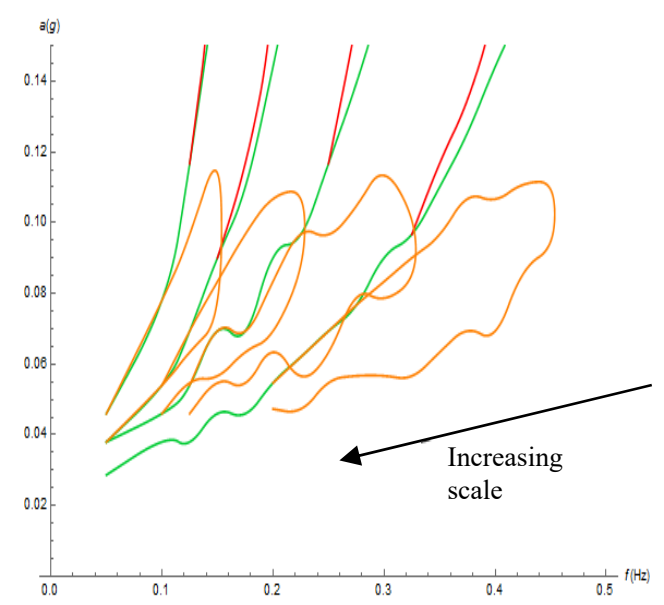

a)

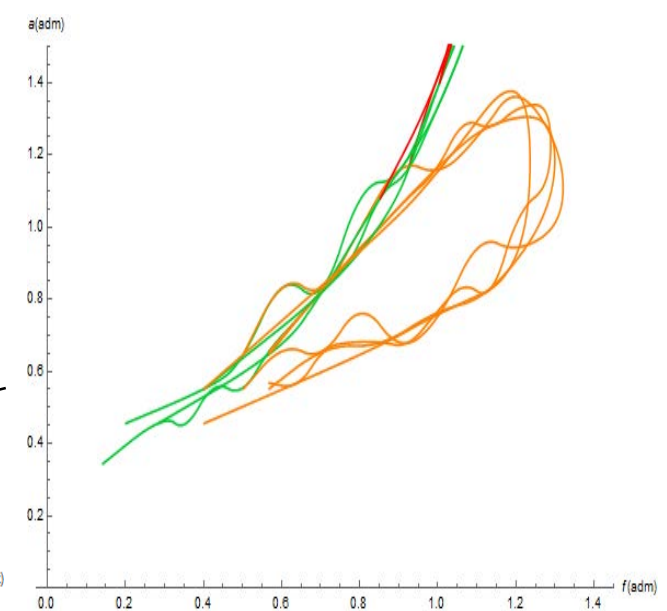

b)

Figure 7: a) Frequency-peak acceleration plots of cosine-like harmonic acceleration pulse of a column with $\mathrm{H} / \mathrm{B}=12,8$ blocks and base diameter variable from 0.25 to $2.00 \mathrm{~m}$; b) adimensionalised plots.

base size has the intent to observe the scale effect on column behaviour. It will therefore be necessary to fix the slenderness of the columns under consideration and vary the base size, and consequently the height to respect the established $\mathrm{H} / \mathrm{B}$ ratio.

The analyses were performed by subjecting a cosine-like harmonic acceleration pulse of the columns having the following characteristics: slenderness $\mathrm{H} / \mathrm{B}=12$; base $\mathrm{B}=0.25-2.00 \mathrm{~m}$; number of blocks $\mathrm{n}_{\mathrm{R}}=8$; internal friction angle $\Phi=30^{\circ}$; damping $=0.5$.

Fig. 6 shows some results of the analyses performed; Fig. 7 shows the same curves but collected in one plot.

The results obtained confirm that larger columns are less vulnerable to collapse by having an increased security area as the column scale increases. It is possible to notice the tendency of the green curves to grow faster as the column scale increases. The yellow curves have the same extension along the vertical axis (they cover a range of extremes $0.04-0.115 \mathrm{~g}$ ), while they have a reduction along the axis of the frequencies as the scale increases. The red curves follow the same pattern as the green ones; they grow faster and appear at frequencies gradually diminishing with increasing the scale.

Again, in this case, by passing to the adimensional parameters, all the curves tend to overlap, showing an invariant behaviour against the scale of the structural element.

\subsection{Safe area obtained by varying the number of the blocks}

Results recorded from analyses carried out on columns composed by a variable number of blocks are presented; the blocks could be only one (case of monolothic columns) up to 16 blocks.

It may be perceived in advance that a column consisting of a small number of blocks is less likely to show partial collapses, limit case is the monoblock column which, by definition, cannot present partial collapses. Increasing the number of blocks will lead to partial collapses 
of different entities at increasing the frequency and the pulse peak acceleration. The analyses were performed by subjecting a cosine-like harmonic acceleration pulse of the columns having the following characteristics: slenderness $\mathrm{H} / \mathrm{B}=12$, base $\mathrm{B}=0.5 \mathrm{~m}$, number of blocks $\mathrm{n}_{\mathrm{R}}$ variable from 1 to 16 , internal friction angle $\Phi=30^{\circ}$, damping $=0.5$. Fig. 8 shows some results of the analyses carried out; in Fig. 9 the same curves are reported but in a unique plot.

Observing the diagrams shown in Fig. 9, it is noticed that increasing the number of blocks the "stability" of the column increases, but some considerations need to be made: the green curves, with the same frequency, show values increasing as the number of blocks reduces (especially for $\mathrm{f}=0.05 \mathrm{~Hz}$, with $\mathrm{n}_{\mathrm{R}}=1$ a collapse acceleration value of $0.086 \mathrm{~g}$ is reached, while with $n_{R}=16$ the value reduces to $0.038 \mathrm{~g}$ ). From this reduction in the stability zone, it can be noticed that increasing the number of blocks the column becomes more susceptible to low frequency collapse phenomena. As the frequencies increase, the behaviour tends to be homologated, making the number of blocks of the column less relevant.

The yellow curves undergo a considerable reduction of their extension in both frequency and acceleration; so it can be inferred that as the number of blocks increases, the column becomes less susceptible to this type of collapse, and that at low frequencies, such phenomenon looks smaller in the columns composed of more blocks.

The red curves are totally absent in the columns with $n_{R}=1$ and $n_{R}=2$ : this is explained by the fact that it is impossible to generate a partial collapse in a monoblock column and it is just as difficult to occur in a column consisting solely of two blocks. Partial collapse occurs starting from the curve with $n_{R}=3$, and it is possible to observe how the increase of the $n_{R}$ parameter significantly decreases the frequency and the peak acceleration beyond such phenomenon occurs.

If in the diagram with $n_{R}=3$ the bifurcation occurs at $f=0.4 \mathrm{~Hz}$ and at $\mathrm{f}=0.344 \mathrm{~g}$, in the diagram with $\mathrm{n}_{\mathrm{R}}=16$, it shows at $\mathrm{f}=0.2 \mathrm{~Hz}$ and at $\mathrm{f}=0.084 \mathrm{~g}$. It can be concluded that as the number of blocks increases, the column becomes more susceptible to partial collapses that occur at lower frequencies and accelerations.

Adimensionalised data return the same trends shown in the previous graphs: this is due to the fact that the variation in the number of blocks does not affect the size of the structure in any way, as it was in the cases discussed in the previous sections.

\subsection{Safe areas obtained by varying the friction coefficient}

As previously mentioned, the model used to describe the mechanical behaviour of the contacts is the Coulomb one with zero cohesion (assuming the blocks in simple support of each other). The following analyses therefore show the variability of the friction angle considered.

The analyses were performed by subjecting a cosine-like harmonic acceleration pulse some columns having the following characteristics: slenderness $H / B=12$, base $B=0.5 \mathrm{~m}$, number of blocks $n_{R}=8$, internal friction angle $\Phi$ variable between $20^{\circ}$ and $40^{\circ}$, damping $=0.5$. Fig. 10 shows the results of the analyses.

It is recorded that the green curves have the same shape and their intercept differs by a quantity of $10-2 \mathrm{~g}$. Also, the size of the areas enclosed by the yellow curves do not differ significantly and the points where they detach from the green curves vary with the same order of magnitude as above. Similar considerations apply to red curves and the bifurcation points where they start. This allows us to state that the friction angle is not a parameter that significantly modifies the impulse response to this structural typology. Similar behaviour is recorded for a sine-like pulse on the columns (Fig. 11). 

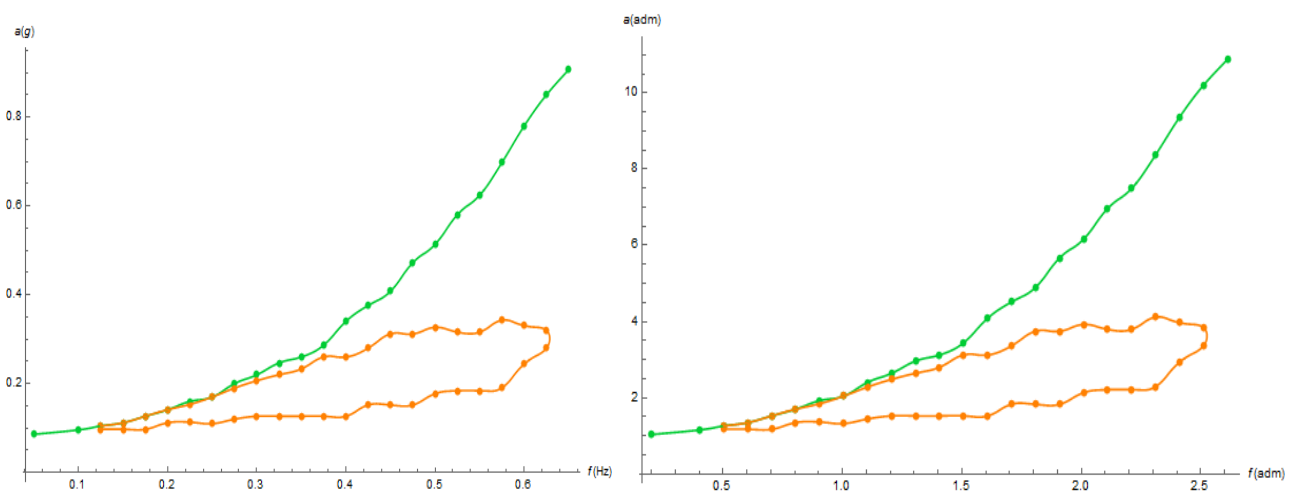

$\mathrm{n}_{\mathrm{R}}=1$

(a)

(b)
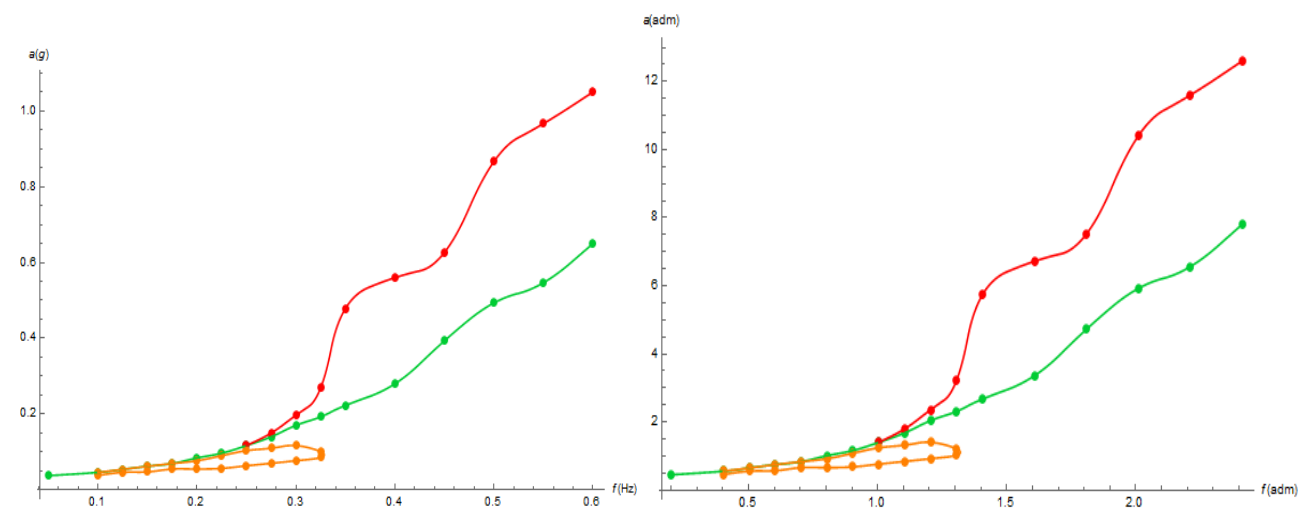

$\mathrm{n}_{\mathrm{R}}=7$

(c)

(d)
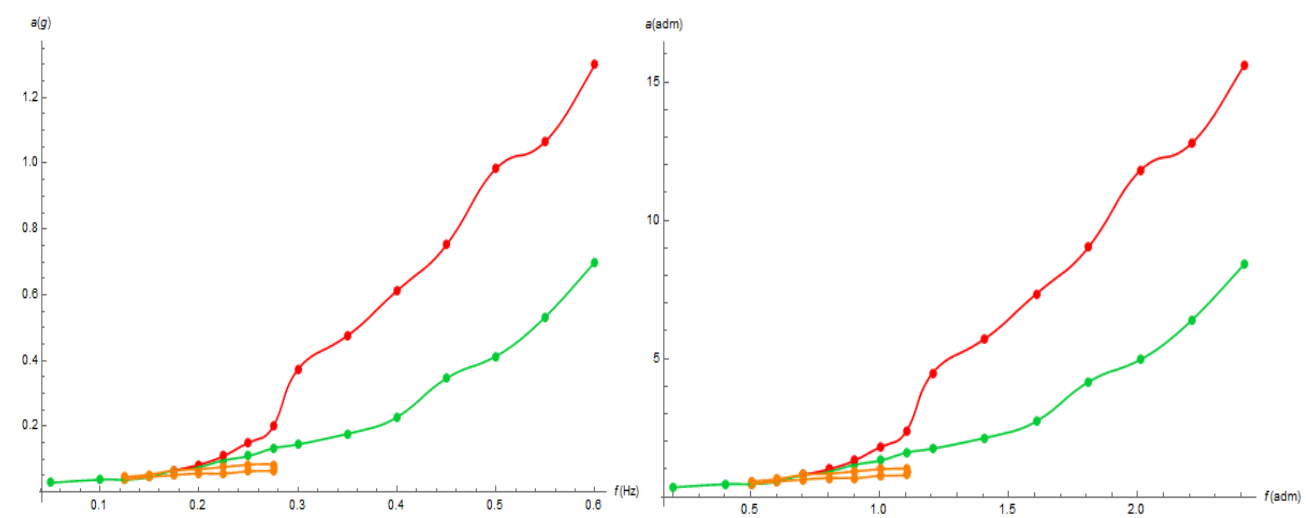

$n_{R}=14$

(e)

(f)

Figure 8: (a), (c), (e) Frequency-peak acceleration plots of the cosine-like harmonic acceleration pulse of a column with $\mathrm{H} / \mathrm{B}=12, \mathrm{~B}=0.5 \mathrm{~m}$ and number of blocks equal to $1,7,14$; (b), (d), (e) adimensionalised plots. 


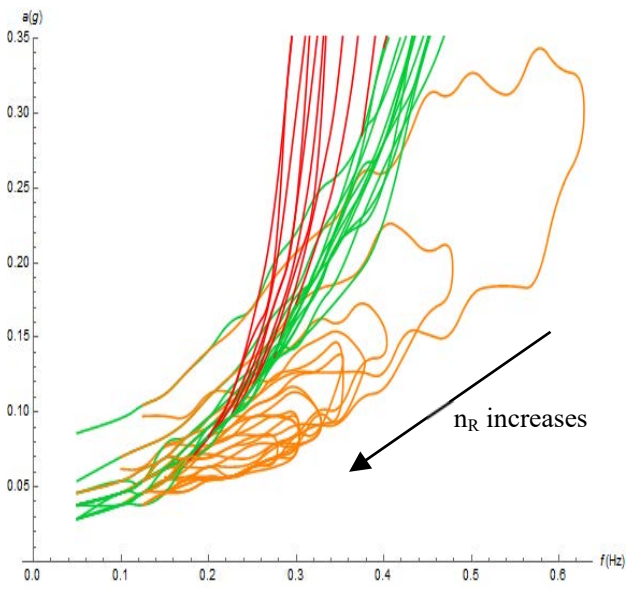

(a)

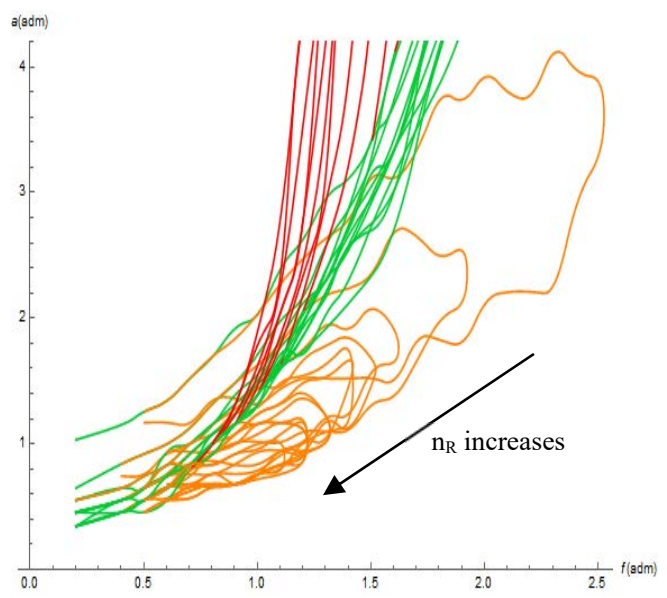

(b)

Figure 9: (a) Frequency-peak acceleration plots of the cosine-like harmonic acceleration pulse of a column with $\mathrm{H} / \mathrm{B}=12, \mathrm{~B}=0.5 \mathrm{~m}$ and number of blocks variable from 1 to 16; (b) adimensionalised plots.

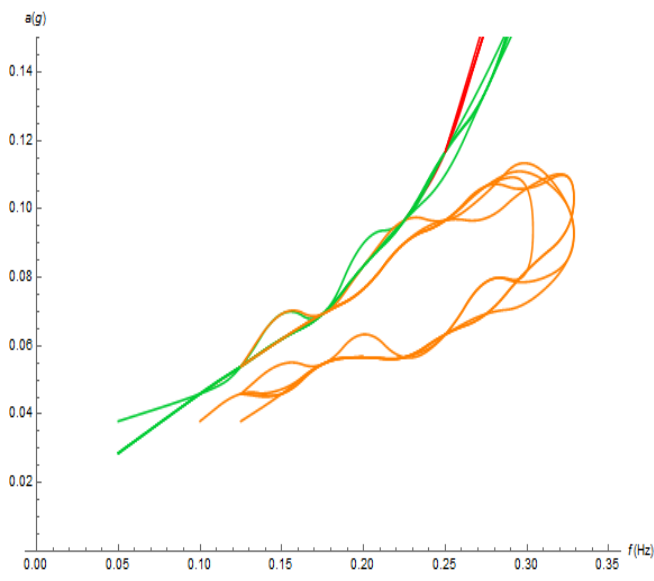

(a)

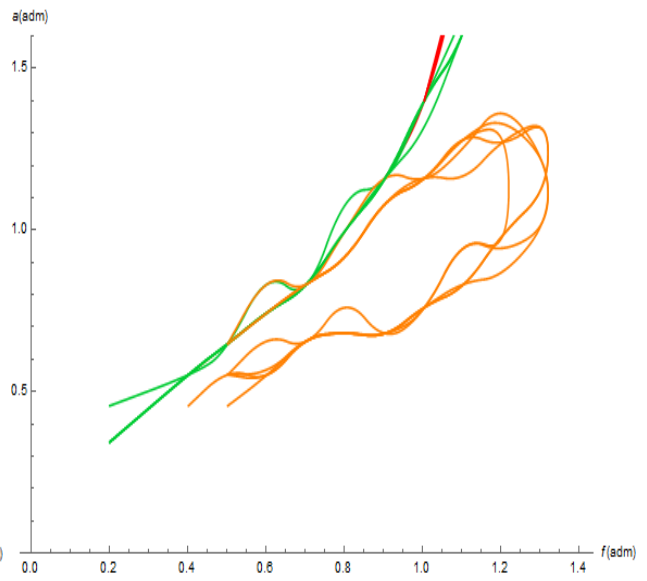

(b)

Figure 10: (a) Frequency-peak acceleration plots of the cosine-like harmonic acceleration pulse of a column with $\mathrm{H} / \mathrm{B}=12, \mathrm{~B}=0.5, \mathrm{n}_{\mathrm{R}}=8$ and $\Phi$ variable between $20^{\circ}$ and $40^{\circ}$; (b) adimensionalised plots.

\section{CONCLUSIONS}

The purpose of this paper was to study the dynamic behaviour of multilayer columns, using the method of the distinct elements (DEM). Starting from the following considerations:

- Multiblock columns do not have a vibration mode (except for small movements);

- Stability is influenced by the variation of the parameters of the structural system; 


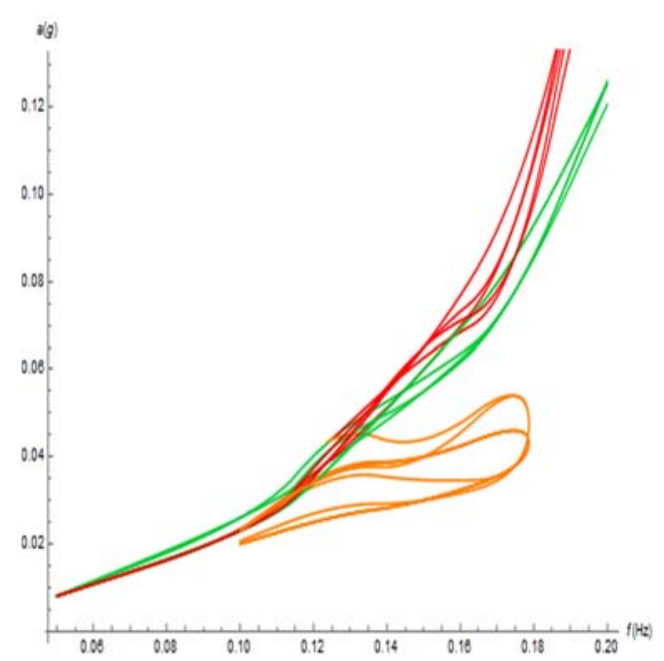

(a)

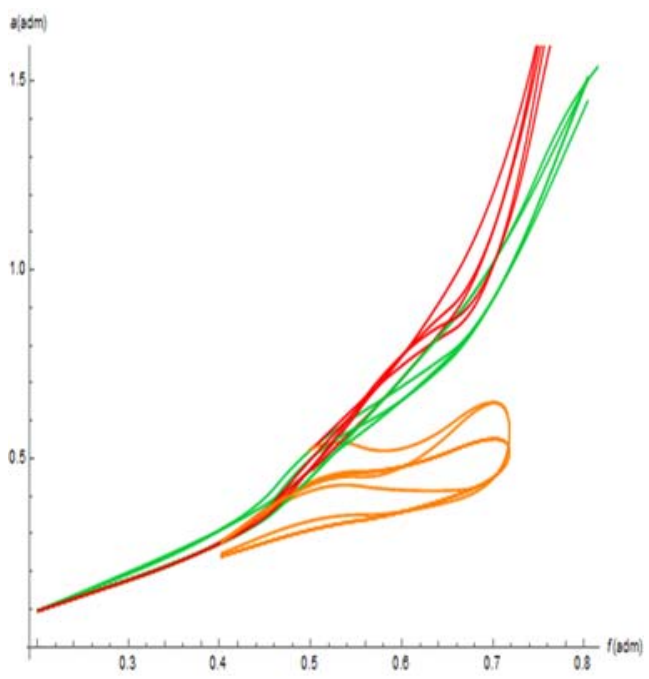

(b)

Figure 11: (a) Frequency-peak acceleration plots of the cosine-like harmonic acceleration pulse of a column with $\mathrm{H} / \mathrm{B}=12, \mathrm{~B}=0.5, \mathrm{n}_{\mathrm{R}}=8$ and $\Phi$ variable between $20^{\circ}$ and $40^{\circ}$; (b) adimensionalised plots.

- The vulnerability of these structures is influenced by the predominant frequency of the earthquake;

- The structural response was observed when the column of cosine and sinusoidal harmonic pulses varies with the slenderness, the effect due to the scale, the number of blocks, the internal friction angle and the damping coefficient.

By analysing the results obtained, it is possible to notice that a slender column at low frequencies is less stable due to the progressive lowering of the detected stability zone. This phenomenon, however, corresponds to a reduction in the collapse area after oscillation of the base line; this evidence suggests that the columns become less and less sensitive to this type of collapse as the H/B ratio increases. Finally, at high frequencies, with the increasing slenderness, an extension of the area where the structure can be defined is established.

Through the study of the variation in scale size, it was possible to conclude that columns with larger dimensions, equal to other parameters such as slenderness, are generally more stable than those of smaller dimensions; the stability zone increases as the scale increases. This phenomenon is associated with the reduction in the area where the collapse is preceded by oscillations, which contributes to the increase in the stability zone in larger columns.

Observing the behaviour shown by the columns varying the number of the blocks, it has been noticed, first, that monoblock structures, or those formed by a few blocks, are not subject to partial collapses. As the number of blocks increases, low-frequency signals are lowered in the stability zone, accompanied by a reduction in the area where the collapse occurs with oscillations: this shows that the column becomes less stable at low frequencies, and therefore more susceptible to collapse without oscillations, but at the same time demonstrates less vulnerability to collapse with oscillations. As the frequencies increase, the 
behaviour of the columns tends to align, making the variability of the number of blocks less influential.

In the analyses conducted, the influence of the friction angle on the overall dynamic behaviour of the columns was also evaluated. As it was intuitive to expect this parameter, it does not cause sensitive changes in the structure's behaviour since the collapses recorded for this kind of stress are rolling and never sliding.

Finally, the influence of the damping coefficient on the global dynamic response was evaluated. At low frequencies, increasing this coefficient, the interface of the stable zone tends to lower, while at high frequencies this trend is reversed, causing the increase in that zone: a higher damping coefficient makes the structure less stable at low frequencies, while at high frequencies it increases its stability. Conversely, assigning a lower damping coefficient it will make the behaviour at low frequencies more stable and vice-versa. The variation of this coefficient also affects the sector where collapse occurs by oscillations; the area that represents it diminishes, in fact, as the damping increases, making the column less subject to this type of instability. From the previous analyses a series of diagrams have been obtained that have stability and collapse zones inside them. The construction of such plots allows predicting the stable behaviour of the structure, knowing the characteristics of the column and the pulse frequency and acceleration of a pulse.

\section{ACKNOWLEDGEMENT}

Italian PRIN 2015 project: "Mitigating the impacts of natural hazards on cultural heritage sites, structures and artefacts (MICHe)" is acknowledged for the support.

\section{REFERENCES}

[1] Lemos, J.V., Discrete element modelling of the seismic behavior of stone masonry arches. Ed. G.N. Pande, J. Middleton, B. Kralj, Proc. of the Fouth Int. Sym. on Computer Methods in Struct. Masonry: London, pp. 220-227, 1998.

[2] Papaloizou, L. \& Komodromos, P., Investigating the seismic response of ancient multi-drum colonnades with two rows of columns using an object-oriented software, Adv. in Eng. Soft., 44(I), pp. 136-149, 2012.

[3] Foti, D., Ivorra, S. \& Vacca, V., In-plane behavior of a masonry stone wall with complex shape blocks. Proc. of the $16^{\text {th }}$ Brick and Block Masonry Conf., IBMAC 2016, Padua, pp. 1587-1591, 2016.

[4] Foti, D., Diaferio, M., Giannoccaro, N.I. \& Ivorra, S., Structural identification and numerical models for slender historical structures. Handbook of research on Seismic Assessment and Rehabilitation of Historic Structures, ed. P. Asteris \& V. Plevris, pp. 674-703, 2015.

[5] Diaferio, M., Foti, D. \& Giannoccaro, N.I., Non-destructive characterization and identification of the modal parameters of an old masonry tower. Proc. EESMS 2014-2014 IEEE Workshop on Environm., Naples, Italy, 2014.

[6] Diaferio, M., Foti, D., Gentile C., Giannoccaro, N.I. \& Saisi, A., Dynamic testing of a historical slender building using accelerometers and radar. Proc. 6th Int. Operation Modal Analysis Conf. IOMAC 2015, Spain, pp. 129-130, 2015.

[7] Bru, D., Ivorra, S., Baeza, F.J., Reynau, R. \& Foti, D., OMA dynamic identification of a masonry chimney with severe cracking condition. Proc. 6th Int. Operation Modal Analysis Conf. IOMAC 2015, Spain, pp. 59-60, 2015.

[8] Foti, D., Dynamic identification techniques to numerically detect the structural damage. The Open Constr. and Build. Tech. J., 7, pp. 43-50, 2013. 
[9] Diaferio, M., Foti, D., Giannoccaro, N.I. \& Ivorra, S., Optimal model through identified frequencies of a masonry building structure with wooden floors. Int. J. of Mech., 8(1), pp. 282-288, 2014.

[10] Cundall, P.A., Computer model for simulating progressive large-scale movements in block rock systems. Proc. Symp. of Intern. Society of Rock Mechanics, 1(II-8), France, 1971.

[11] ITASCA Consulting Group, 3DEC Version 5.0: User's Guide, Minneapolis, Minnesota, 2013.

[12] Rocco, G., Guida alla lettura degli ordini architettonici antichi - i. lo ionico, Liguori Editore, Maggio, 2003.

[13] Zang, J. \& Makris, N., Rocking response of free-standing blocks under cycloidal pulses. ASCE J. of Eng. Mech., 127(5), pp. 473-483, 2001. 